\title{
Phosphatidylglycerol of rat lung
}

\section{Intracellular sites of formation de novo and acyl species pattern in mitochondria, microsomes and surfactant}

\author{
Michael SCHLAME, $\dagger$ Bernd RÜSTOW, ${ }^{*}$ Dietrich KUNZE, ${ }^{*}$ Harald RABE* and Gunter REICHMANN* \\ *Institute of Pathological and Clinical Biochemistry and †Department of Anaesthesiology and Intensive Care Medicine, \\ Charité Hospital, Humboldt University, 1040 Berlin, German Democratic Republic
}

\begin{abstract}
The subcellular site of phosphatidylglycerol (PG) formation for lung surfactant has not been convincingly clarified. To approach this problem we analysed the acyl species pattern of lung PG in mitochondria, microsomes and surfactant by h.p.l.c. separation of its 1,2-diacyl-3-naphthylurethane derivatives. Both mitochondrial and microsomal PG proved identical with surfactant PG, containing the major species 1-palmitoyl-2-oleoyl-PG and 1,2-dipalmitoyl-PG. The fatty acid composition of mitochondrial PG differs markedly from that of diphosphatidylglycerol. This may be taken as an indication that mitochondrial PG is synthesized on purpose to form surfactant, rather than being only the precursor of diphosphatidylglycerol. In vitro, sn-[U-14 C]glycerol 3-phosphate incorporation into PG of mitochondria or microsomes occurs in the presence of CTP, ATP and CoA but independently of the supply of exogenous lipoidic precursors. Although the rate in vitro of autonomous PG synthesis, and the endogenous PG content, are higher in mitochondria than in microsomes, it is assumed that both subcellular fractions are involved in PG formation for surfactant.
\end{abstract}

\section{INTRODUCTION}

PG is a major phospholipid of plants (Roughan \& Slack, 1984) and bacteria (Cronan, 1978) whereas the lung is the only mammalian tissue containing PG in significant amounts (Hallman \& Gluck, 1974). In lung, PG is an essential constituent of the surfactant material, demonstrated by the fact that PG deficiency is accompanied by respiratory distress syndrome (Liau et al., 1984; Mitnick et al., 1980). Accordingly, lung tissue has a high PG turnover rate (Frosolono, 1977) which is likely a metabolic property of type II pneumócytes, the cells of surfactant formation and secretion. In other mammalian organs PG is found in trace amounts only. It is localized in mitochondria where it serves as intermediate of DPG generation. An enhancement of DPG synthesis at expense of PG secretion during acute alveolar injury (Liau et al., 1984) seems to confirm the involvement of mitochondrial PG metabolism for surfactant production. However, it is a feature of lung that glycerophosphate phosphatidyltransferase (EC 2.7.8.5) is also present in microsomes even if mitochondrial activity is significantly higher (Hallman \& Gluck, 1975; Mavis \& Vang, 1981). The consideration of lung microsomes being the primary site of PG formation was suggested by the competition between phosphatidylinositol and PG synthesis in lung (Hallman \& Epstein, 1980) and type II pneumocytes (Batenburg et al., 1985; Bleasdale et al., 1983).

This paper reports a further attempt to investigate the subcellular site of surfactant PG formation by evaluating the PG acyl species in mitochondria, microsomes and surfactant. The species pattern, used as intrinsic marker, is nearly identical in these fractions but sharp differences between the fatty acid patterns of mitochondrial PG and DPG become obvious. To our knowledge, this is the first report of the complete molecular species distribution in mammalian PG, whereas that of plant PG species has already been published (Norman et al., 1984).

Until now it has not been shown whether mitochondria can synthesize PG autonomously via their own snglycerol 3-phosphate acylation or if they depend upon supply of lipoidic precursors (PA or CDP-diacylglycerol) from microsomes, as suggested by Stuhne-Sekalec \& Stanacev (1980). Here we show that both rat lung mitochondria and microsomes can perform formation de novo of PG autonomously.

\section{EXPERIMENTAL}

\section{Chemicals}

ATP, CTP, CoA, phospholipase C (Bacillus cereus), succinate and cytochrome $c$ were obtained from Boehringer Mannheim, and lipid standard substances were purchased from Sigma Chemical Co. except for PG which was produced as described by Bergelson (1980). Hepes and Florisil were from Serva, $s n-\left[\mathrm{U}-{ }^{14} \mathrm{C}\right] \mathrm{glycerol}$ 3-phosphate (specific radioactivity $170 \mathrm{mCi} / \mathrm{mmol}$ ) from The Radiochemical Centre, Amersham, 2,2'-p-phenylbis-(5-phenyloxazole) from Riedel-de-Haen AG Seelze (Hannover, Germany), 2,5-diphenyloxazole from Packard-Becker B.V. Chemical Co. (Groningen, The Netherlands) and silica gel HR from Merck. Dimethylformamide was purified by distillation with octadecylisocyanate. $\alpha$-Naphthylisocyanate was purified by distillation in vacuo. All other chemicals were commercially obtained in analytical grade. 


\section{Isolation of subcellular fractions and surfactant}

Rat lung subcellular fractions (from male Wistar rats) were isolated at $2{ }^{\circ} \mathrm{C}$ in a medium containing sucrose $(0.15 \mathrm{~mol} / 1), \mathrm{KCl}(50 \mathrm{mmol} / 1)$, Hepes $(20 \mathrm{mmol} / \mathrm{l})$ (pH 7.4), EDTA (1 mmol/l) and 2-mercaptoethanol $(2 \mathrm{mmol} / \mathrm{l})$ by a slight modification of the isolation procedure published by Evans \& Scholz (1975). Rats (body wt. 200-250 g) were anaesthetized with diethyl ether and killed by opening the neck vessels. After cannulating the right ventricle, lungs were perfused with isolation medium. Five lungs were minced in $50 \mathrm{ml}$ of isolation medium and homogenized using a loosefitting Teflon/glass Potter-Elvehjem homogenizer (100 rev./min). The homogenate was centrifuged at $600 \mathrm{~g}$ for $20 \mathrm{~min}$. Crude mitochondria were pelleted out of the supernatant by centrifugation at $9000 \mathrm{~g}$ for $20 \mathrm{~min}$. After careful removal of the fluffy layer, resuspended mitochondria were pelleted by a short-term ( $4 \mathrm{~min}$ ) centrifugation step at $15000 \mathrm{~g}$. The final pellet (about $2 \mathrm{mg}$ of protein per rat) was obtained by further centrifugation of the resuspended mitochondria at $15000 \mathrm{~g}$ for $10 \mathrm{~min}$. Microsomes were pelleted out of the post$9000 \mathrm{~g}$ supernatant by centrifugation at $100000 \mathrm{~g}$ for $90 \mathrm{~min}$.

Rat lung alveolar wash was obtained by five successive lavages with $\mathrm{NaCl}(0.15 \mathrm{~mol} / \mathrm{l}) /$ Tris $(10 \mathrm{mmol} / \mathrm{l})$ (pH 7.5) via the cannulated trachea. Before lavage the lungs were degassed by vacuum. The alveolar wash was cleared of intact cells and tissue pieces by centrifugation at $600 \mathrm{~g}$ for $30 \mathrm{~min}$. Surfactant material in the remaining supernatant was pelleted by centrifugation at $100000 \mathrm{~g}$ for $1 \mathrm{~h}$ at $4^{\circ} \mathrm{C}$ and subjected to lipid extraction. Phospholipid pattern of this pellet revealed $82 \%$ phosphatidylcholine, 9\% PG, 3\% phosphatidylethanolamine, $2 \%$ sphingomyelin, $1 \%$ phosphatidylserine and $3 \%$ unidentified phospholipids, in agreement with surfactant phospholipid composition (Possmayer et al., 1984).

\section{sn-[U-14 C]Glycerol 3-phosphate incorporation into PG}

The complete assay for PG synthesis contained either lung mitochondria or microsomes corresponding to $0.4 \mathrm{mg}$ of protein $/ 0.2 \mathrm{ml}, \mathrm{KCl}(0.15 \mathrm{~mol} / 1)$, Hepes $(50 \mathrm{mmol} / \mathrm{l})$ (pH 7.8), cysteine (10 mmol/1), EDTA (1 mmol/l), magnesium acetate $(3 \mathrm{mmol} / \mathrm{l})$, ATP (4 mmol/l), CTP $(1 \mathrm{mmol} / 1)$, CoA (0.17 mmol $/ 1)$ and sn-[U-14C]glycerol 3-phosphate $(0.5 \mathrm{mmol} / \mathrm{l})$ (specific radioactivity 5000 d.p.m./nmol). Incubation at $37^{\circ} \mathrm{C}$ was started by addition of subcellular preparations and was stopped by lipid extraction.

\section{Lipid analysis}

Lipids were extracted according to Bligh \& Dyer (1959). Separation by two-dimensional t.l.c. was performed on silica gel $\mathrm{HR} /$ Florisil $(99: 1, \mathrm{w} / \mathrm{w})$ with chloroform/methanol/25\% $\mathrm{NH}_{3} /$ water (180:108:11:11, by vol.) in the first direction and chloroform/acetone/methanol/acetic acid/water $(10: 4: 2: 2: 1$, by vol.) in the second direction. Spots, identified by co-chromatography with standard lipids, were scraped off for scintillation counting or determination of phosphate according to Hallermayer \& Neupert (1974). Measurement of radioactivity was carried out with a Packard liquid-scintillation spectrometer (model 3380). Silica gel spots were suspended in $8 \mathrm{ml}$ of dioxane/water $(5: 1, \mathrm{v} / \mathrm{v})$ with diphenyloxazole
( $5.8 \mathrm{mg} / \mathrm{ml}), 2,2^{\prime}$ - $p$-phenylbis-(5-phenyloxazole) $(0.25 \mathrm{mg} / \mathrm{ml})$ and naphthalene $(83 \mathrm{mg} / \mathrm{ml})$.

\section{H.p.l.c. analysis of PG molecular acyl species}

This h.p.l.c. separation procedure was published by Krüger et al. (1984). PG was isolated by t.l.c. and extracted from the silica gel spots with chloroform $/$ methanol $(2: 1, \mathrm{v} / \mathrm{v})$. After evaporation, $50-200 \mathrm{nmol}$ of PG were redissolved in $0.5 \mathrm{ml}$ of diethyl ether/ethanol $(98: 2$, $\mathrm{v} / \mathrm{v})$ and mixed with $0.1 \mathrm{ml}$ of Hepes $(0.25 \mathrm{~mol} / \mathrm{l})$ (pH 5.5) containing 5 i.u. of phospholipase C (B. cereus). Incubation at $20^{\circ} \mathrm{C}$ for $12 \mathrm{~h}$ revealed complete hydrolysis to diacylglycerol which was extracted in chloroform/ methanol $(2: 1, \mathrm{v} / \mathrm{v})$. After evaporation to dryness the diacylglycerols were divided in $20-50 \mathrm{nmol}$ portions and redissolved in dimethylformamide. Derivatization was carried out at $85^{\circ} \mathrm{C}$ for $2 \mathrm{~h}$ with a 200 -fold molar excess of $\alpha$-naphthylisocyanate in the presence of a 4-fold molar excess of 1,4-diazabicyclo(2,2,2)octane. Excess reagent was destroyed by addition of methanol. The h.p.l.c. separation was carried out on a Hewlett-Packard model 1084 B equipped with a Fluorichrom detector (Varian, Los Altos, CA, U.S.A.; excitation $280 \mathrm{~nm}$, emission $360 \mathrm{~nm})$. RP $18(5 \mu \mathrm{m})$ h.p.l.c. columns (length $150 \mathrm{~mm}$, diameter $3.2 \mathrm{~mm}$ ) were eluted with acetonitrile/water by use of a linear gradient with decreasing water content starting from acetonitrile/water $(83: 17$, v/v). H.p.l.c. peaks were identified by gas chromatographic analysis of the methyl esters of the fatty acids which may be released from the diacylglycerol derivatives. Linear dependence on the $s n$-1,2-dipalmitoylglycerol content of the peak area could be demonstrated in a range from 0.2 to 50 nmol (see Krüger et al., 1984).

\section{Other methods}

Protein was determined with the method of Lowry et al. (1951). Activity of succinate:cytochrome $c$ reductase was recorded at $550 \mathrm{~nm}$ by incubating mitochondria or microsomes $(0.05 \mathrm{mg}$ of protein $/ \mathrm{ml})$, cytochrome $c$ from horse heart $(1 \mathrm{mg} / \mathrm{ml}), \mathrm{KCl}$ (60 mmol/1), sucrose (0.1 mol/l), $\mathrm{MgCl}_{2}(10 \mathrm{mmol} / \mathrm{l})$, succinate $(5 \mathrm{mmol} / \mathrm{l})$ and $\mathrm{KCN}(2 \mathrm{mmol} / \mathrm{l})$ at $20^{\circ} \mathrm{C}$. Cytochrome $a$ content (Rieske, 1967) and NADPH : cytochrome $c$ reductase activity (Sottocasa et al., 1967) were determined as described.

\section{RESULTS}

Fig. 1 shows $s n-\left[\mathrm{U}-{ }^{14} \mathrm{C}\right]$ glycerol 3-phosphate incorporation into mitochondrial PG as a function of time. Because no lipoidic precursor was added these data reflect the complete formation de novo of $\mathrm{PG}$ and/or the incorporation of the glycerophosphate head group using endogenous mitochondrial precursors. Radioactivity was distributed among PA (about 50\%), PG (10-20\%), diacylglycerol (10-20\%), monoacylglycerol, triacylglycerol and phosphatidylcholine (together 10-20\%) but no labelling of DPG and phosphatidylethanolamine could be observed.

If fatty acid activation was prevented by omitting ATP and $\mathrm{CoA}$ the $s n-\left[\mathrm{U}-{ }^{14} \mathrm{C}\right]$ glycerol 3-phosphate incorporation into $P G$ was significantly reduced to $18.6 \%$ (S.E.M. $=15.1, n=3$ ) of the initial activity (Fig. 1). The fact that PA synthesized de novo was a suitable substrate for mitochondrial PG generation was directly demon- 


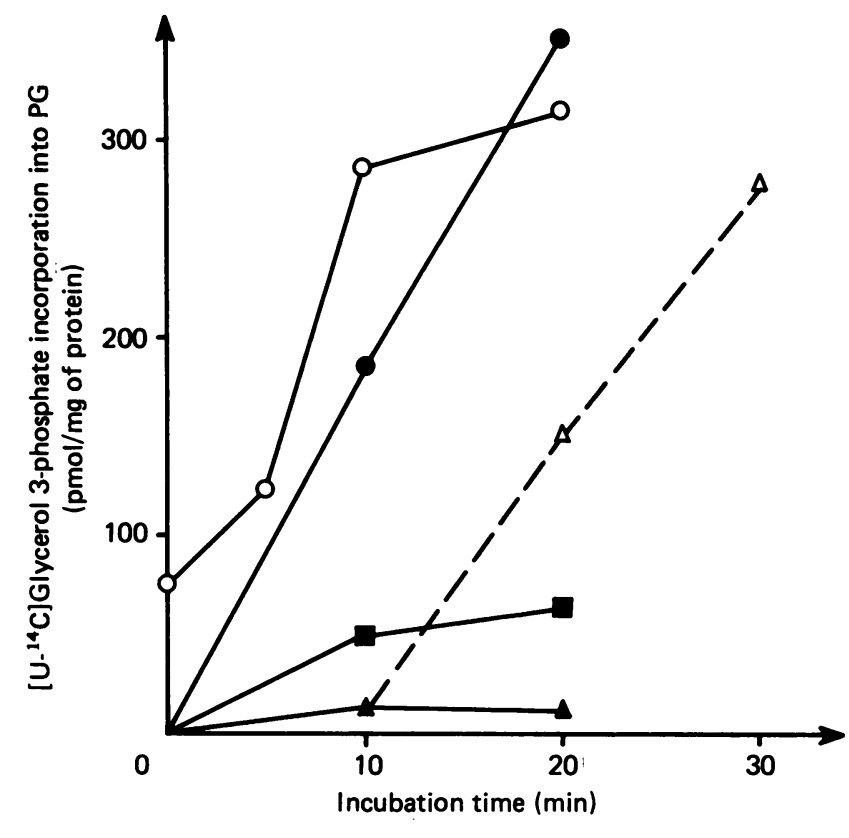

Fig. 1. Synthesis de novo of PG by rat lung mitochondria in vitro

$s n-\left[\mathrm{U}-{ }^{14} \mathrm{C}\right]$ Glycerol 3-phosphate incorporation into PG was measured in a complete assay $(O)$, in the absence of ATP and CoA ( $\square)$, or in the absence of CTP $(\boldsymbol{\Delta})$. Points on the broken line $(\triangle)$ were obtained after subsequent addition of CTP $(1 \mathrm{mmol} / \mathrm{l})$ to a portion of the assay without CTP. In a further approach $(O)$, mitochondria were incubated in the absence of CTP for $15 \mathrm{~min}$, yielding $6.6 \mathrm{nmol}$ of $s n$-[U-14 C]glycerol 3-phosphate/mg of protein $(85 \%$ of total lipid radioactivity) in PA. Mitochondria were washed, repelleted and reincubated in a complete assay for PG synthesis except that $\boldsymbol{s n}$-glycerol 3-phosphate was not labelled. strated by the conversion of prelabelled mitochondrial $\left[{ }^{14} \mathrm{C}\right] \mathrm{PA}$ to PG (open circles in Fig. 1).

Furthermore, PG labelling depended on CTP (Fig. 1) whose absence decreased the incorporation of ${ }^{14} \mathrm{C}$ radioactivity to $8.4 \%$ (S.E.M. $=4.2, n=3$ ). Subsequent addition of CTP completely restored the synthesis of PG (broken line in Fig. 1). Dependency upon CTP of mitochondrial PG formation means that almost no endogenous CDP-diacylglycerol was available in lung mitochondria.

In Table $1\left[{ }^{14} \mathrm{C}\right] \mathrm{PG}$ formation in rat lung mitochondria is compared with that of rat lung microsomes in three independent experiments in which both subcellular fractions were derived from the same lung homogenate respectively. All individual experiments revealed a higher synthesis de novo of $\mathrm{PG}$ in mitochondria than in microsomes. This seemed not to be caused by a more active $s n$-glycerol 3-phosphate acylation in mitochondria because the ratio of mitochondrial $\left[{ }^{14} \mathrm{C}\right] \mathrm{PG} /\left[{ }^{14} \mathrm{C}\right] \mathrm{PA}$ was significantly higher. The distribution of PG-generating capacities between lung mitochondria and microsomes was paralleled by the PG content in the corresponding fractions (Table 1). On the basis of marker enzyme distribution (microsomal marker, NADPH:cytochrome $c$ reductase), microsomes should contaminate the mitochondrial preparation by less than $20 \%$. Vice versa, the microsomal preparation contains not more than $10 \%$ mitochondrial membranes (mitochondrial markers, succinate: cytochrome $c$ reductase and cytochrome $a+a_{3}$ ). To estimate the lamellar body contamination mitochondria were subjected to upward flotation density gradient centrifugation according to Chander et al. (1983), revealing a sharp turbid band of lamellar bodies at $0.3-0.5 \mathrm{~mol}$ of sucrose/1, marked by its high lipid/protein mass ratio. This fraction contained $10 \%$ of total phospholipids of the preparation whereas $60 \%$ of phospholipids cosedimented with heavy mitochondria

Table 1. Rate of PG formation de novo and PG content in rat lung mitochondria and microsomes

Rat lung mitochondria and microsomes were incubated in a complete assay for PG synthesis for 10 or $20 \mathrm{~min}$. $\left[{ }^{14} \mathrm{C}\right] \mathrm{PG} /\left[{ }^{14} \mathrm{C}\right] \mathrm{PA}$ ratio and PG content are means of experiments 1-3. S.E.M. $(n=3)$ is given in parentheses.

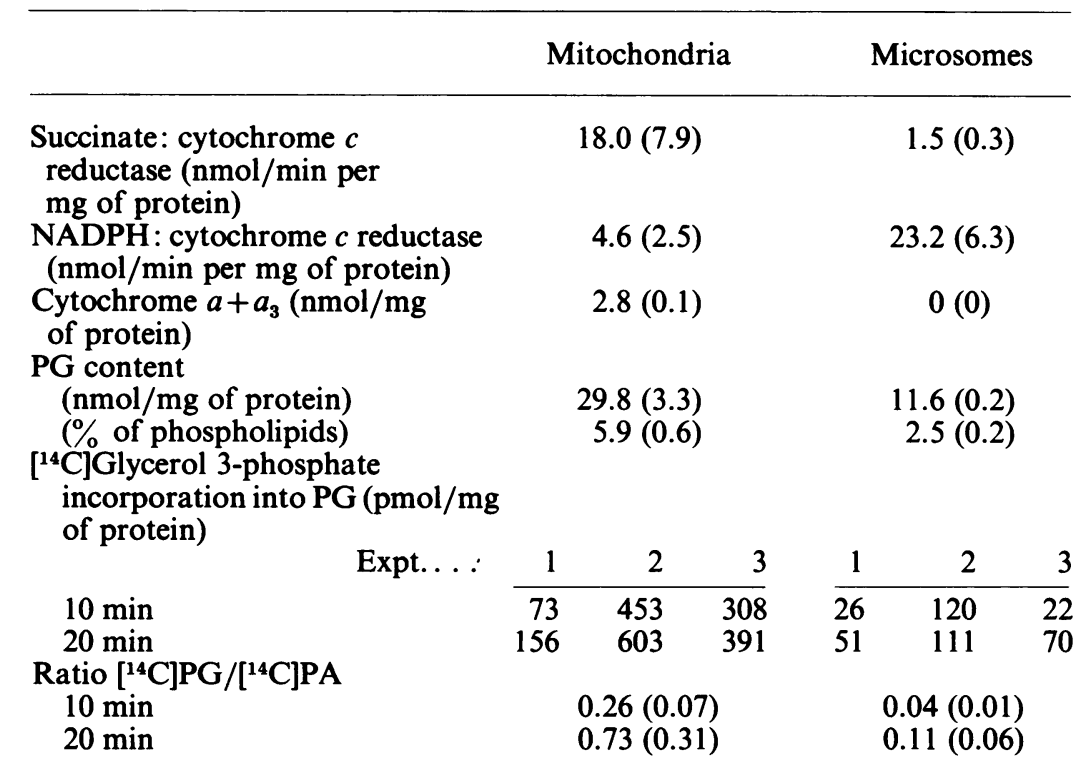


Table 2. Molecular species of PG from rat lung fractions

Values are means, with S.E.M. (if $n=3$ ) or range (if $n=2$ ) in parentheses, of the percentage distribution. For peak numbers

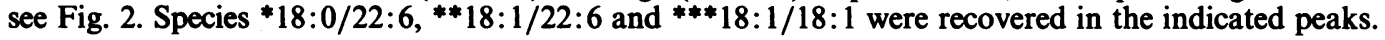

\begin{tabular}{lcccc}
\hline $\begin{array}{l}\text { Molecular } \\
\text { species }\end{array}$ & $\begin{array}{c}\text { Peak } \\
\text { no. }\end{array}$ & $\begin{array}{c}\text { Surfactant } \\
(n=2)\end{array}$ & $\begin{array}{c}\text { Microsomes } \\
(n=3)\end{array}$ & $\begin{array}{c}\text { Mitochondria } \\
(n=3)\end{array}$ \\
\hline $16: 0 / 16: 0$ & 8 & $16.9(5.3)$ & $17.7(2.3)$ & $21.0(4.2)$ \\
$16: 0 / 18: 1^{*}$ & 9 & $26.7(2.1)$ & $25.2(0.5)$ & $28.2(0.8)$ \\
$16: 0 / 18: 2$ & 4 & $9.8(0.3)$ & $9.5(0.3)$ & $11.9(0.4)$ \\
$16: 0 / 20: 3$ & 7 & $1.2(0.5)$ & $1.3(0.2)$ & $0.6(0.5)$ \\
$16: 0 / 20: 4^{* *}$ & 3 & $11.2(3.1)$ & $10.5(1.3)$ & $8.5(1.3)$ \\
$16: 0 / 22: 6$ & 1 & $10.6(2.1)$ & $8.5(1.3)$ & $10.0(2.0)$ \\
$16: 0 / 18: 0$ & 15 & $1.4(0.2)$ & $2.8(0.3)$ & $1.3(0.1)$ \\
$18: 0 / 18: 1$ & 16 & $3.9(0.4)$ & $4.8(1.0)$ & $2.9(0.5)$ \\
$18: 0 / 18: 2$ & 11 & $2.0(0.3)$ & $2.9(0.3)$ & $2.0(0.5)$ \\
$18: 0 / 20: 3$ & 14 & $1.1(0.3)$ & $1.1(0.2)$ & $0.4(0.3)$ \\
$18: 0 / 20: 4^{* * *}$ & 10 & $1.7(0.1)$ & $3.7(0.5)$ & $3.5(1.9)$ \\
$18: 0 / 22: 5$ & 12 & $1.6(0.2)$ & $1.6(0.7)$ & $1.0(0.7)$ \\
Not identified & & $11.9(4.4)$ & $10.1(0.9)$ & $8.8(0.8)$ \\
\end{tabular}

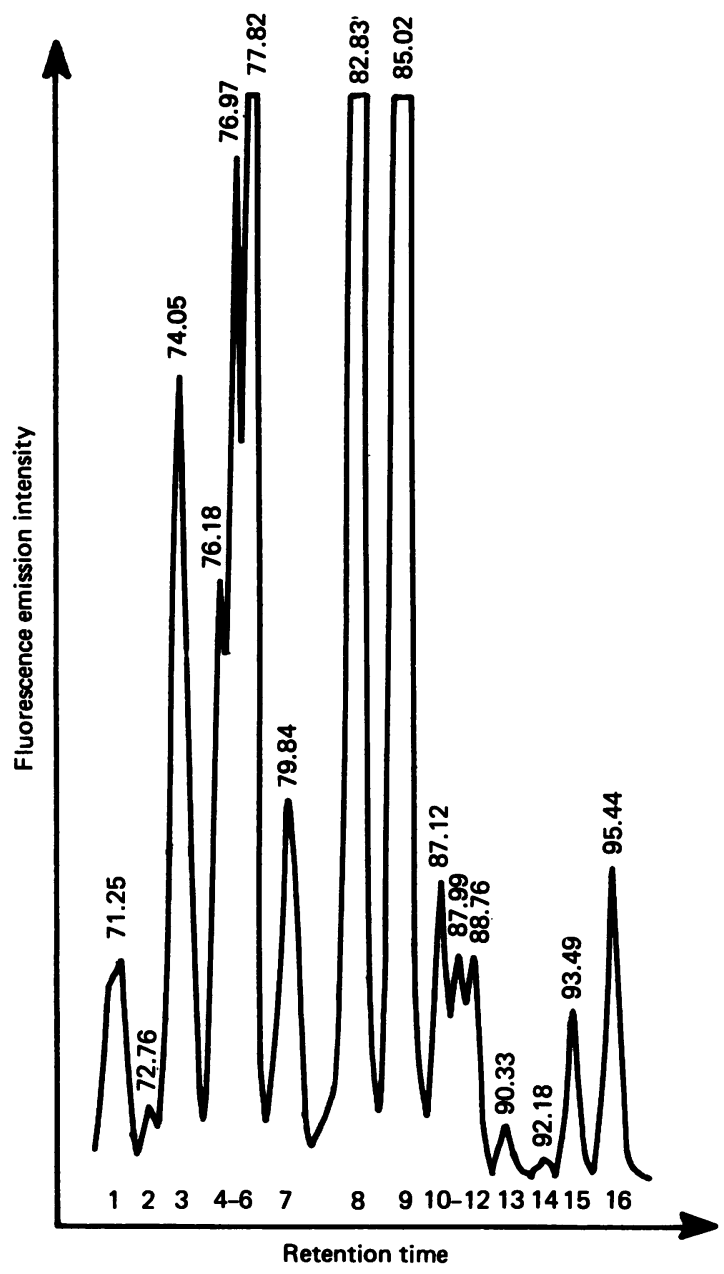

Fig. 2. Chromatogram of $P G$ molecular species from rat lung mitochondria

Retention times (min) are given on top of peaks, which refer to species listed in Table 2. (pellet fraction in the density gradient) and $30 \%$ of phospholipids were distributed among the other zones of the gradient, representing light mitochondria, disrupted mitochondrial membranes and other contaminating subcellular fractions.

Table 2 shows the molecular species pattern of PG from mitochondria, microsomes and surfactant, which did not prove different. In all preparations 16:0/18:1 and 16:0/16:0 represented the main species, together accounting for $40-45 \%$. A further $25-30 \%$ of PG species consisted of 1-palmitoyl-2-polyunsaturated molecules $(16: 0 / 18: 2,16: 0 / 20: 4$ and 16:0/22:6). To demonstrate the resolution of the acyl species derived from $P G$, one h.p.l.c. profile is depicted in Fig. 2. Because this h.p.l.c.

Table 3. Fatty acid composition of DPG and PG from rat lung mitochondria

After isolation of DPG by t.l.c. its fatty acids were converted to methyl esters (according to Morrison \& Smith, 1964) and analysed with a Varian 2100 gas chromatograph using a coiled glass column with $10 \%$ EGSS-X (for details see Krüger et al., 1984). PG fatty acid profile was calculated from Table 2 . Abbreviation: n.i., not identified.

\begin{tabular}{|c|c|c|}
\hline \multirow{2}{*}{$\begin{array}{l}\text { Fatty } \\
\text { acid }\end{array}$} & \multicolumn{2}{|c|}{$\begin{array}{c}\text { Composition } \\
(\%)\end{array}$} \\
\hline & DPG & PG \\
\hline $16: 0$ & 7.8 & 56.2 \\
\hline $16: 1$ & 9.7 & n.i. \\
\hline $18: 0$ & 3.8 & 5.4 \\
\hline $18: 1$ & 22.7 & 17.3 \\
\hline $18: 2$ & 35.9 & 7.7 \\
\hline $20: 3$ & 1.9 & 0.6 \\
\hline $20: 4$ & 4.1 & 6.7 \\
\hline $22: 5$ & 6.9 & 0.6 \\
\hline $22: 6$ & 2.4 & 5.6 \\
\hline
\end{tabular}


technique could not be applied to DPG a comparison between DPG and PG was performed on the basis of their fatty acid patterns (Table 3). DPG of rat lung mitochondria showed a preference for oleic and linoleic acids, as reported for mitochondrial DPG from other sources (Keenan et al., 1970; Wood \& Harlow, 1969). In mitochondrial PG of lung, these fatty acids were largely replaced by palmitate. The high palmitate content of PG from lung has already been reported by Sanders \& Longmore (1975).

\section{DISCUSSION}

The intracellular site of PG synthesis for lung surfactant is a matter of controversial discussion stressing either the mitochondrial (Mavis \& Vang, 1981) or the microsomal (Hallman \& Gluck, 1974, 1975) importance, respectively. Although the microsomal rate of $s n$-glycerol 3-phosphate acylation is higher than the mitochondrial one, our experiments indicate that mitochondria are more active in PG formation de novo via PA (Table 1). This is in line with the distribution of endogenous PG content as well as glycerophosphate phosphatidyltransferase activity between lung mitochondria and microsomes (Mavis \& Vang, 1981). On the other hand it was stated that the microsomal capability of PG formation is an intrinsic activity and cannot be related to mitochondrial contamination (Bleasdale \& Johnston, 1982; Hallman \& Gluck, 1974, 1975). This, in principle, was confirmed by the presented data. If one compares the [U-14 C]glycerol 3-phosphate incorporation into PG of mitochondria and microsomes for each experiment individually (see six pairs of values given in Table 1), the microsomal rate is $23.0 \%$ (s.E.M. $=9.6$ ) of the mitochondrial rate of incorporation. Considering a mitochondrial contamination in microsomes of about $10 \%$, it seems more likely that rat lung microsomes perform their own independent PG formation de novo. However, with respect to the fluctuations between different preparations, these data should be interpreted with some caution. That the high PG content of mitochondria is not due to contamination by lamellar bodies may be estimated by the low percentage of lamellar body phospholipids in the mitochondrial preparation.

Although mitochondrial generation of PA de novo is generally recognized, it is not yet evident if mitochondrial PA is a suitable substrate for polyglycerophosphatide formation. Our data imply that PG synthesis of rat lung mitochondria occurs independently of lipid precursors generated in other subcellular sites. Because the absence of ATP and CoA or CTP is accompanied by a nearly complete loss of PG formation in mitochondria, it is considered that mitochondrial fatty acid activation as well as conversion of mitochondrial PA to CDPdiacylglycerol are indispensable steps in the formation of $\mathbf{P G}$ in lung mitochondria (Fig. 1). In contrast, Stuhne-Sekalec \& Stanacev (1979) reported that the PG synthesis of liver mitochondria in vitro is controlled by exogenous supply of CDP-diacylglycerol. The question arises whether or not autonomous PG formation is a specialized mitochondrial function present only in lung. Further it remains unknown what kind of lung cells are responsible for this function and if it has implications for the production of surfactant.

Mitochondrial PG is thought to be the immediate pre- cursor of DPG synthesis. However, no $s n-\left[\mathrm{U}-{ }^{14} \mathrm{C}\right] \mathrm{glycerol}$ 3-phosphate incorporation into DPG could be observed under the conditions described. It is not yet clear if this is due to insufficient concentration of divalent cations, which are crucial cofactors of DPG synthesis (Hostetler et al., 1975), or if PG generated de novo is not available for DPG synthetase.

Considering PG as the direct precursor of DPG, their fatty acid profiles should be quite close, since no satisfactory acyl selectivity could be established, neither for conversion of PG to DPG (Hostetler et al., 1975) nor for the reacylation of lyso-DPG (Eichberg, 1974). The sharp difference between the fatty acid patterns of mitochondrial PG and DPG recorded in Table 3 makes it likely that, in lung, mitochondrial PG has more physiological significance than being only a lipid intermediate on the DPG biosynthetic path.

This significance might be an involvement in surfactant generation. The correspondence of diacyl species of PG from mitochondria, microsomes and surfactant (Table 2) is taken as an indication that mitochondrial as well as microsomal PG could be secreted into the surfactant layer. This result contradicts the observation of Hallman \& Gluck (1975) that mitochondrial PG contains less palmitate than microsomal and surfactant PG. It remains a matter of speculation what function the proposed bilocal distribution of PG formation might have for surfactant metabolism.

Surfactant PG shows a heterogenous range of different acyl combinations (Table 2), whereas surfactant phosphatidylcholine consists mainly of disaturated species (Possmayer et al., 1984). Probably PG stabilizes surfactant by its specific head group but does not require a high percentage of disaturated acyl moieties. This suggestion is supported by Shelley et al. (1984), who reported a high variability of PG fatty acid patterns among different animal species.

\section{REFERENCES}

Batenburg, J. J., Klazinga, W. \& van Golde, L. M. G. (1985) Biochim. Biophys. Acta 833, 17-24

Bergelson, L. D. (1980) Lipid Biochemical Preparations, pp. 195-198, Elsevier/North Holland Biomedical Press, Amsterdam, New York, Oxford

Bleasdale, J. E. \& Johnston, J. M. (1982) Biochim. Biophys. Acta 710, 377-390

Bleasdale, J. E., Tyler, N. E., Busch, F. N. \& Quirk, J. G. (1983) Biochem. J. 212, 811-818

Bligh, E. G. \& Dyer, W. J. (1959) Can. J. Biochem. 37, 911-917

Chander, A., Dodia, C. R., Gil, J. \& Fisher, A. B. (1983) Biochim. Biophys. Acta 753, 119-129

Cronan, J. E. (1978) Annu. Rev. Biochem. 47, 163-195

Eichberg, J. (1974) J. Biol. Chem. 249, 3423-3429

Evans, R. M. \& Scholz, R. W. (1975) Biochim. Biophys. Acta 381, 278-291

Frosolono, M. F. (1977) in Lipid Metabolism in Mammals (Snyder, F., ed.), vol. 2, pp. 1-38, Plenum Press, New York, London

Hallermayer, G. \& Neupert, W. (1974) Hoppe-Seyler's Z. Physiol. Chem. 355, 279-288

Hallman, M. \& Epstein, B. L. (1980) Biochem. Biophys. Res. Commun. 92, 1151-1159

Hallman, M. \& Gluck, L. (1974) Biochem. Biophys. Res. Commun. 60, 1-7

Hallman, M. \& Gluck, L. (1975) Biochim. Biophys. Acta 409, 172-191 
Hostetler, K. Y., Galesloot, J. M., Boer, P. \& van den Bosch, H. (1975) Biochim. Biophys. Acta 380, 382-389

Keenan, T. W., Awasthi, Y. C. \& Crane, F. L. (1970) Biochem. Biophys. Res. Commun. 40, 1102-1103

Krüger, J., Rabe, H., Reichmann, G. \& Rüstow, B. (1984) J. Chromatogr. 307, 387-392

Liau, D. F., Barrett, C. R., Bell, A. L. L., Cernansky, G. \& Ryan, S. F. (1984) J. Lipid Res. 25, 678-683

Lowry, O. H., Rosebrough, N. J., Farr, A. L. \& Randall, R. J. (1951) J. Biol. Chem. 193, 265-275

Mavis, R. D. \& Vang, M. J. (1981) Biochim. Biophys. Acta 664, 409-415

Mitnick, M. A., De Marco, B. \& Gibbson, J. M. (1980) Clin. Chem. 26, 277-281

Morrison, W. R. \& Smith, M. J. (1964) J. Lipid Res. 5, 600-605

Norman, H. A., McMillan, C. \& Thompson, G. A. (1984) Plant Cell Physiol. 25, 1437-1444
Possmayer, F., Yu, S.-H., Weber, J. M. \& Harding, P. G. R. (1984) Can. J. Biochem. Cell Biol. 62, 1121-1133

Rieske, J. S. (1967) Methods Enzymol. 10, 488-493

Roughan, G. \& Slack, R. (1984) Trends Biochem. Sci. 9, 383-386

Sanders, R. L. \& Longmore, W. J. (1975) Biochemistry 14, 835-840

Shelley, S. A., Paciga, J. E. \& Balis, J. U. (1984) Lipids 19, 857-862

Sottocasa, G. L., Kuylenstierna, B., Ernster, L. \& Bergstrand, A. (1967) J. Cell Biol. 32, 415-438

Stuhne-Sekalec, L. \& Stanacev, N. Z. (1979) Can. J. Biochem. 57, 618-624

Stuhne-Sekalec, L. \& Stanacev, N. Z. (1980) Can. J. Biochem. 58, 1082-1090

Wood, R. \& Harlow, R. D. (1969) Arch. Biochem. Biophys. 135, 272-281

Received 7 April 1986/10 July 1986; accepted 6 August 1986 